\title{
Gefühle ins Recht setzen: Wann sind politische Emotionen (noch) demokratisch?
}

\section{Einleitung}

Als im März 2014 eine überwältigende Mehrheit der russischen Bürger die Annexion der Krim durch ihren Präsidenten Wladimir Putin bejubelte, wurde die Differenz zur politischen Kultur in den meisten westlichen Demokratien wie in einem Brennglas sichtbar. „Warum freut Ihr Euch nicht ebenso mit uns über die Rückkehr der Krim zu Russland wie wir uns mit Euch über die deutsche Wiedervereinigung gefreut haben?", fragten russische Bürger. Während im Falle der Wiedervereinigung Deutschlands der Ausdruck politischer Gefühle nicht nur als kompatibel mit den Werten der Demokratie, sondern als selbst genuin demokratisch gewertet wurde, herrschte unter den westlichen Beobachtern ein Konsens dahingehend, dass der Jubel russischer Bürger über die Annexion der Krim einen neuen, nun triumphalistisch zur Schau gestellten Nationalismus sichtbar machte.

Obwohl sich viele offensichtliche Differenzen zwischen den beiden Beispielen nennen lassen, ist die allgemeine Antwort darauf, wann politische Gefühle (noch) als demokratisch zu werten sind, alles andere als einfach. Vielmehr scheint die Frage, unter welchen Bedingungen politische Gefühle mit den normativen Ansprüchen an ein demokratisches Regierungssystem und eine demokratische politische Kultur vereinbar sind, auch aus Sicht der politischen Theorie hochkomplex. Sie ist nicht nur abhängig vom Demokratiebegriff, ${ }^{1}$ sondern scheint von verschiedenen theoretischen Standpunkten aus unterschiedliche Arten der Beantwortung zu erfahren.

Dass eine emotionsfreie Politik undenkbar ist, darf heute hingegen als Konsens vorausgesetzt werden. Die Hoffnung auf eine von allen irrationalen Gefühlen gereinigte, rein expertokratisch ausgerichtete oder von gänzlich rational gestimmten Bürgern hervorgebrachte Politik, in deren Willensbildungsprozessen ausschließlich Argumente Gehör finden, darf heute als vergangene Utopie gelten. Nicht nur die Empirie politischer Kommunikation widerspricht systematisch diesem Ideal; auch und vor allem die Ergebnisse der Emotionsforschung aus anderen Teildisziplinen wie der Hirnforschung, der Verhaltensökonomie oder der Philosophie haben den

1 Zur Differenz zwischen einer liberalen Skepsis gegenüber politischen Gefühlen und einer republikanischen Affirmation staatlicher Gefühlsproduktion vergleiche Schaal/Heidenreich 2013. 
Dualismus von Vernunft und Gefühl relativiert. Die Einsicht, dass Entscheidungen nie ausschließlich rational fallen, dass Rationalität und Emotionalität bestenfalls Dimensionen, nicht aber Gegensätze von mentalen Zuständen und damit auch politischen Entscheidungen bezeichnen, wird heute kaum noch bestritten. Im Gegenteil: Die Rhetorik der Sachlichkeit und Eindeutigkeit, wonach es an der Zeit sei, nun doch endlich einmal an ,unbestreitbare Tatsachen“ oder ,eindeutige Sachlagen“ zu erinnern, sieht sich nach den kollektiven Erfahrungen, die gerade die deutschen Bürger in den Diskussionen über die friedliche Nutzung der Atomkraft oder die Finanz- und Schuldenkrise gesammelt haben, in der Defensive befindlich. Alle politischen Fragen sind immer schon in ein „framing“ gesetzt und daher mit emotionalen Konnotationen und Abschattungen umgeben; einen ganz und gar sachlichen Blick auf die Dinge außerhalb jeder Rhetorik kann es aus dieser Perspektive in der Politik nicht geben.

Doch der Konsens, dass eine Politik ohne Gefühle undenkbar ist, verschiebt den Fokus der Debatte nur auf die nachgelagerte Frage, welche Formen der Emotionalität einerseits demokratiegefährdend und welche Formen demokratischer Leidenschaft andererseits funktionsnotwendig sind. Im Folgenden sollen drei idealtypische Antworten diskutiert werden. Eine erste mögliche Antwort verweist auf die Inhalte der politischen Gefühle. Aus dieser Perspektive gibt es demokratische und weniger demokratische Gefühle: Hass und Angst gefährden aus dieser Sichtweise Demokratien, während Empörung, Empathie oder gar Liebe zu tragenden Elementen einer demokratischen Kultur erklärt werden. Diese Sichtweise ist sehr weit verbreitet, kann aber, wie der folgende Abschnitt zeigen wird, wichtige prinzipielle Einwände schwer widerlegen (2.). Ein zweiter idealtypischer Vorschlag zielt auf die Frage der Authentizität politischer Gefühle: Totalitäre, autoritäre oder defekte Demokratien zeichnen sich aus dieser Sicht durch die systematische Herstellung politischer Gefühle durch Eliten aus. Undemokratisch sind politische Gefühle vor allem dann, wenn sie unauthentisch sind, also systematisch durch eine Staatsführung hervorgebracht und kanalisiert werden. Der Begriff der „Emotionalisierung“, der in diesem Kontext oft Verwendung findet, impliziert eine manipulative Absicht. Auch hier zeigt eine genauere Betrachtung, dass diese Sichtweise in der Anwendung auf unüberwindbare Schwierigkeiten stößt (3.). Eine dritte idealtypische Antwort verweist auf den Bearbeitungsmodus, den Gefühle in der politischen Arena erfahren. Empörung, Wut, Begeisterung, Stolz - all diese emotionalen Ressourcen sind aus dieser Perspektive als Ausgangsmaterial einer Sublimierung, Reflexion und Strukturierung von politischen Gefühlen auch in Demokratien legitim, müssen sich jedoch dem Prozess einer demokratischen Rechtfertigung unterziehen und in diesem bewähren. Weder Inhalt noch Ursprung von politischen Gefühlen sind folglich ent- 
Gefühle ins Recht setzen: Wann sind politische Emotionen (noch) demokratisch?

scheidend, sondern der Status, der politischen Gefühlen zugeschrieben wird - ultimativer Ersatz für Argumente oder zu überprüfender Ausgangspunkt der Debatte entscheidet über ihre demokratische oder undemokratische Wirkung (4.). In der Rekonstruktion dieser drei Idealtypen werden die behandelten Theoretiker die Funktion illustrativer Beispiele einnehmen, da eine maßstabsgetreue Rekonstruktion der Debatte über politische Gefühle in diesem Rahmen nicht möglich ist. Ein Schlussabschnitt wird der Frage nachgehen, welche Konsequenzen sich aus einer entsprechenden Sicht auf die politischen Prozesse ergeben.

\section{Auf den Inhalt kommt es an: Demokratische und undemokratische Gefühle}

Die erste idealtypische Antwort bei der Frage nach der Differenz zwischen demokratischen und undemokratischen politischen Gefühlen verweist auf die Inhalte. In vielen Fällen wird die Differenz eher implizit benannt und bestimmte Gefühle eher assoziativ mit demokratischen Idealen in Verbindung gebracht. Das zentrale Argument lautet, dass es Gefühle, die zur Kooperation und Solidarität, und andere, die zu Egoismus und Rücksichtslosigkeit anleiten, gibt. Für die erste Sorte werden meist Liebe, Empathie oder Empörung genannt; typische Beispiele für undemokratische oder demokratiegefährdende Gefühle sind entsprechend Angst, Wut, Hass.

Klassische Beispiele für diesen Typus von Argumentation finden wir bei Judith Shklar, die vor allem auf die Bedeutung der Empörung für den demokratischen Prozess hingewiesen hat (Shklar 1997), oder bei Martha Nussbaum, die in ihrem neusten Buch „Political Emotions“ ganz explizit die Liebe als demokratisches Gefühl feiert und zur systematischen Produktion entsprechender Emotionen durch eine gezielte Politik des ,shaping compassion“ (Nussbaum 2013: 257 ff.) rät. Auch außerhalb des engeren Feldes der Politischen Theorie finden sich entsprechende Argumentationsmuster. So hat beispielsweise Naomi Klein auf die aus ihrer Sicht demokratiegfährdenden Effekte von Furcht hingewiesen: Wo die Bürger verängstigt seien und das kognitiv-rationale Denken vom Gefühl des Bedroht-Seins gelähmt werde, ließen sich zweifelhafte politische Entscheidungen sehr viel leichter durchsetzen (Klein 2007).

Die inhaltliche Differenzierung nach demokratischen und undemokratischen Gefühlen scheint auf den ersten Blick einleuchtend. In der Tat lassen sich totalitäre Systeme als Formen einer systematischen Herstellung und Bewirtschaftung von Gefühlen wie Hass, Ressentiment oder Angst verstehen. Vor allem Hass lässt sich mit den Idealen der Demokratie und den demokratischen Lebensform kaum in Einklang bringen: Auch Demokraten haben zwar Gegner, aber eben keine Feinde, die sie hassen könnten oder müssten. Zugleich setzt das Funktionieren eines demokra- 
tischen Regierungssystems und das Gedeihen einer demokratischen Kultur ein Höchstmaß an Kooperationswillen und Kompromissfähigkeit voraus. Beide Kompetenzen sind ohne eine entwickelte Empathiefähigkeit schwer denkbar. Auch erleben wir in Demokratien zahllose Appelle an die Solidarität der Bürger untereinander, an Mitgefühl und Einfühlungsvermögen. Feierveranstaltungen und historische Gedenkstunden bieten in Demokratien die Möglichkeit, derartige Gefühle zu pflegen und in Inszenierungen hervorzurufen, wobei Symbolen eine besondere Rolle als Medium emotionaler Integration zukommt (Göhler 2012).

Bei genauerer Betrachtung scheint eine inhaltliche Differenzierung jedoch problematisch. Ob Gefühle demokratische Strukturen stützen und offene Prozesse eher fördern, entscheidet sich wohl nicht nur an ihrer inhaltlichen Ausrichtung. All zu verlockend ist die Option, positive Gefühle mit der Demokratie zu assoziieren, negative mit Autokratien oder Diktaturen. „Liebe“ mag in Form des Patriotismus vor allem in der republikanischen Tradition des politischen Denkens eine genuin demokratische Tugend sein - doch die Übergänge zu einer nicht mehr demokratischen Form blinder Vaterlandsliebe bleiben fließend. Liebe kann auch blind machen für Rechtsverstöße (,Right or wrong - my country!“) und zur systematischen Ungerechtigkeit anleiten. Zu den zentralen Errungenschaften funktional ausdifferenzierter Gesellschaften gehört die Unterscheidung von Recht und Moral. Gerade vermeintlich positive Gefühle und Haltungen wie Liebe oder Solidarität können den Anspruch auf formale Gleichheit in Rechtssystemen auch in einem negativen Sinne untergraben. Da Liebe dazu anleitet, Ausnahmen zuzulassen, kann sie zur Gefahr von Rechtsstaatlichkeit werden.

Ebenso lassen sich weniger diffuse emotionale Einstellungen wie Solidarität missbrauchen, um soziale Konflikte zu kaschieren oder falsche Opferbilder zu verbreiten. Die Solidarität einer In-Group geht zwangsläufig auf Kosten einer OutGroup; dieser Mechanismus ist normativ zunächst unbestimmt und kann sowohl demokratische als auch undemokratische Formen annehmen. In zahllosen Fällen wird der Rechtsbruch (gerade des internationalen Rechts) mit der Pflicht zur Solidarität mit (angeblich oder tatsächlich) bedrohten Minderheiten begründet. Das Spektrum politischer Gefühle lässt sich folglich nicht einfach nach den Polen demokratisch/undemokratisch sortieren.

\section{Von „oben“ gemachte Gefühle vs. authentischer bottom-up-Prozess}

In der Erforschung politischer Gefühle bildet die Produktion politischer Gefühle in totalitären Systemen zweifellos den Sonderfall eines breit verhandelten Themas: Die systematische Herstellung von Ressentiments, Mißtrauen und Hass gegen Min- 
Gefühle ins Recht setzen: Wann sind politische Emotionen (noch) demokratisch?

derheiten und vor allem Juden ist in der Totalitarismus- und Antisemitismusforschung in all ihren Facetten untersucht. Gerade vor dem Hintergrund unseres breiten Wissens über die Manipulierbarkeit von politischen Gefühlen durch Propaganda bietet sich eine Unterscheidung nach dem Ursprung von politischen Gefühlen an. Entscheidend ist nach dieser zweiten idealtypischen Perspektive weniger der Inhalt politischer Gefühle als vielmehr ihr Entstehungsprozess: Entstehen sie aus dem politischen Konflikt selbst, aus authentischen Erfahrungen der Bürger, so bilden sie einen zentralen Indikator für Veränderungspotenziale. Entstehen sie jedoch als Produkt einer gezielten politischen Strategie durch Eliten, so bleiben sie ideologisch und werden schlimmstenfalls zur Bedrohung der Demokratie. Verordnete Liebe (zum Vaterland oder zur politischen Führung), geschürte Empörung und Wut (gegen einen inneren oder äußeren Feind), ja selbst die staatlich erzwungene Freundschaft (zum Beispiel zu „Brudervölkern“) sind aus dieser liberalen Perspektive abschreckende Beispiele für eine Emotionalisierung der Bevölkerung durch Eliten. Auch alle Versuche, positive Gefühle wie Glück bei den Bürgern herzustellen oder systematisch zu ermöglichen, stehen aus dieser Perspektive im Verdacht, den Lebens- und Glücksentwürfen der Bürger auf undemokratische Weise vorauszugreifen und eine letztlich totalitäre „Volksbeglückung“ zu beabsichtigen.

Die normative Forderung, die sich aus dieser Perspektive zumindest implizit ergibt, muss auf einen emotional neutralen Staat abstellen. In der Tat war diese Hoffnung mit der neuzeitlichen Vorstellung einer Privatisierung der Idee des ,guten Lebens" verbunden: Die politische Auseinandersetzung sollte mit Argumenten und Interessen, nicht aber mit Leidenschaften gespeist werden (vgl. die klassische Studie von Hirschman 1987).

Fraglich bleibt an dieser These, ob sich die Authentizität von Gefühlen tatsächlich feststellen lässt. Selbstverständlich gibt es eindeutige Fälle eines durch Propaganda herbeigeführten „Volkszorns“ oder einer medial induzierten Massenhysterie. Doch in der Regel werden sich individuelle und soziale Aspekte von Gefühlen nicht sauber trennen lassen. Ähnlich wie im Falle der Sprache ist auch unser Gefühlsleben immer schon sozial vermittelt und erlernt. Es orientiert sich mimetisch oder in Abgrenzung an Rollenmustern, Vorbildern, medialen und politischen Idealen. Die Vorstellung ,authentischer" Gefühle wirkt vor diesem Hintergrund beinahe wie das Relikt einer romantischen Subjektivitätsemphase. Bürger und politische Eliten interagieren in der Produktion politischer Gefühle wie zwei Tänzer, die sensibel auf die Signale des jeweils anderen reagieren, diese mal verstärken, mal ihnen entgegenwirken. Entsprechend gehört die Frage zu den klassischen Topoi der politischen Auseinandersetzung, ob die Thematisierung gewisser Probleme nun eine berechtigte Furcht von Bürgern aufgreift oder bloß eine diffuse Angst (bspw. vor Einwanderern) schürt 
- eine Frage, die nicht von einem archimedischen Punkt aus eindeutig zu beantworten ist.

Der zentrale Einwand gegen die zweite idealtypische Antwort auf die Frage nach der Differenz zwischen demokratischen und undemokratischen politischen Gefühlen zielt auf die hier vorausgesetzte Konzeption von Kausalität. Selbst in so vermeintlich augenscheinlichen Fällen wie der propagandistischen Produktion des antisemitischen Hasses wird man eher von komplexen Wechselwirkungen zwischen Eliten und Bürgern ausgehen müssen. Zwar ist es richtig, dass dieser Hass durch Propagandaschriften wie „Die Protokolle der Weisen von Zion“ bewusst geschürt wurde; zugleich musste der Rezeptionsboden eines diffusen christlichen Antijudaismus gegeben sein, um entsprechende Wirkungen zu entfalten. Es scheint daher äußerst zweifelhaft, authentische Gefühle der Empörung (wenn es sie denn gibt) als a priori legitimen Ausdruck von Missachtungserfahrungen zu werten, auf die das politische System responsiv zu antworten hätte. Auch die vermeintlich authentische Wut von „Wutbürgern“ kann unbegründet sein und zu demokratiegefährdendem Handeln anleiten.

\section{Gefühle - Motor oder Lenker des demokratischen Prozesses}

Eine dritte idealtypische Perspektive akzentuiert weder den Inhalt noch den Ursprung politischer Gefühle, sondern vielmehr ihren Status in der politischen Öffentlichkeit. Die entscheidende Frage lautet hier: Dienen politische Gefühle der Immunisierung von politischen Positionen, der Verkürzung oder Verhinderung von Debatten, der „Legitimation durch Gefühl“ anstelle von Verfahren - oder unterwerfen sie sich selbst dem Rechtfertigungs- und Transformationsdruck, dem auch Argumente in der demokratischen Öffentlichkeit ausgesetzt sind? Demokratisch wären politische Gefühle aus dieser Perspektive genau dann, wenn sie als Ausgangspunkt, Motor oder Antreiber politischer Auseinandersetzungen fungieren, ohne diese mit dem Verweis auf Unbegründbarkeit für beendet zu erklären.

Die oft verwendete Hintergrundmetaphorik dieser Vorstellung ist alt und verweist nicht zuletzt auf das platonische Bild vom Wagenlenker, der die stürmischen Rösser des Gefühls zu lenken wissen muss. Doch die modernen Vorstellungen eines in Empörung ihren Ausgangspunkt findenden „Kampfes um Anerkennung“(Honneth) gehen über die platonische Konzeption weit hinaus, weil sie dessen letztlich naturrechtlichen Rahmen sprengen. Während es bei Platon noch um die Annäherung an ein gegebenes Ideal (die Idee des Guten) geht, konzipieren moderne republikanische und deliberative Theorien den Prozess der Sublimierung und Reflexion politischer 
Gefühle als einen ergebnisoffenen Konstruktionsprozess, in dem die soziale und politische Ordnung nicht abgebildet, sondern geschaffen wird.

Vor allem bei Jürgen Habermas und Axel Honneth lässt sich eine Konsequenz dieser Position deutlich beobachten, nämlich die zentrale Bedeutung des Rechts als Medium der Kanalisierung und Sublimierung von Gefühlen, ja der Transformation emotionaler Äußerungen in Argumente und schließlich Institutionen. Auch wenn sich bei beiden Autoren bei genauerer Betrachtung deutlich verschiedene Akzente abzeichnen, Habermas über Metaphern wie „Schleuse“ oder „Filter“ stärker die Exklusion von Gefühlen (vgl. hierzu Weber 2012), Honneth stärker die Transformation von Gefühlen vor Augen zu haben scheint, so bildet das Recht doch in beiden Theorien den entscheidenden Faktor einer Strukturierung bisweilen diffuser emotionaler Impulse. Auch authentische politische Emotionen berechtigen aus dieser Perspektive nicht zum Rechtsbruch; vielmehr zwingt die politische Auseinandersetzung, die auf eine Gesetzgebung abzielt, dazu, die Inhalte, Quellen und Ziele politischer Emotionen explizit zu machen und so aus eher diffusen Affekten transparente und gefestigte Einstellungen werden zu lassen.

\section{Schlussbemerkung}

Der Durchgang durch drei idealtypische Versuche einer systematischen Unterscheidung zwischen demokratischen und undemokratischen politischen Gefühlen erlaubt es, die Ambivalenz politischer Gefühle genauer zu verstehen. Ein Begriff wie „Wutbürger“ eignet sich zur Prüfung dreier idealtypischer Antworten. Wut kann nicht a priori für demokratisch erklärt oder von einer genuin demokratischen „Empörung" unterschieden werden. Auch stellt die Authenzitität der Wut oder Empörung des Wutbürgers ein nicht ausreichendes Kriterium dar. Zwar wird ein mit den Mitteln der Propaganda im top-down-Verfahren hervorgerufener „Volkszorn“ undemokratisch bleiben. Doch das Spektrum der Emotionalisierungen kennt auch subtilere Formen der Steuerung von Emotionen, die nicht a priori undemokratisch sind, sondern zum Spiel politischer Auseinandersetzung gehören. Die Unterscheidung zwischen authentischer Empörung engagierter Bürger und der gesteuerten Wut populistisch manipulierter „Wutbürger“ mag daher analytisch fruchtbar sein, dürfte sich aber als kaum operationalisierbar erweisen - nicht zuletzt, weil Menschen dazu neigen, die eigenen Gefühle für authentisch zu halten.

Bietet die dritte hier vorgeschlagene Unterscheidung eventuell ein hilfreiches Kriterium? Die Wut der Wutbürger wäre demnach demokratisch, wenn und so lange sie sich der Forderung einer öffentlichen Rechtfertigung stellt und bereit ist, $\mathrm{zu}$ erläutern, aus welchen Gründen und gegen wen sie sich richtet. Als Motor und An- 
treiber politischer Auseinandersetzung mag sie den demokratischen Willensbildungsprozess fördern, kann ihn jedoch niemals ersetzen. Politische Emotionen können sich - analog zu politischen Argumenten - in der politischen Auseinandersetzung als unbegründet und daher „falsch“ erweisen. Folgt man dieser Argumentation, so stellt sich der Prozess der politischen Auseinandersetzung nicht nur als ein Ringen um Interessen und Argumente dar, sondern auch als ein Prozess der Produktion, Reflexion und Zivilisierung von Gefühlen. Demokratie verlangt von den Bürgern daher nicht nur eine Distanz gegenüber den eigenen kognitiven Überzeugungen, die Bereitschaft, dazuzulernen, sondern auch einen reifen Umgang mit den eigenen politischen Emotionen. Politische Emotionen ins Recht zu setzen, bedeutet dann sowohl, ihre Bedeutung anzuerkennen wie auch ihre Einhegung und Reflexion durch das Recht zu betreiben.

\section{Literatur}

Göhler, Gerhard, 2012: Die affektive Dimension der Demokratie - Überlegungen zum Verhältnis von Deliberation und Symbolizität, in: Felix Heidenreich/Gary S. Schaal (Hrsg.), Politische Theorie und Emotionen, Baden-Baden, 235-253. Hall, Cheryl A., 2005: The Trouble with Passion: Political Theory Beyond the Reign of Reason, New York.

Heidenreich, Felix, 2012: Versuch eines Überblicks: Politische Theorie und Emotionen, in: Felix Heidenreich/Gary S. Schaal (Hrsg.), Politische Theorie und Emotionen, Baden-Baden, 9-26.

Hirschman, Albert O., 1987: Leidenschaften und Interessen, Frankfurt a. M.

Honneth, Axel, 2011: Das Recht der Freiheit, Berlin.

Klein, Naomi, 2007: Die Schock-Strategie: Der Aufstieg des Katastrophen-Kapitalismus, Frankfurt a. M.

Nussbaum, Martha, 2013: Political Emotions: Why Love Matters for Justice, Princeton.

Schaal, Gary S./Heidenreich, Felix, 2013: Politik der Gefühle: Zur Rolle von Emotionen in der Demokratie, in: APuZ 32-33/2013, 3-11.

Shklar, Judith, 1997: Über Ungerechtigkeit. Erkundungen zu einem moralischen Gefühl, Frankfurt a. M.

Weber, Florian, 2012: Unterkühlter Diskurs. Zum Verhältnis von Emotionen und Deliberation bei Jürgen Habermas, in: Felix Heidenreich/Gary S. Schaal (Hrsg.), Politische Theorie und Emotionen, Baden-Baden, 199-215. 
Gefühle ins Recht setzen: Wann sind politische Emotionen (noch) demokratisch?

Korrespondenzanschrift:

Dr. Felix Heidenreich

Internationales Zentrum für Kultur- und Technikforschung

Universität Stuttgart

Geschwister-Scholl-Str. 24

70174 Stuttgart

E-Mail: felix.heidenreich@izkt.uni-stuttgart.de 